\title{
Infarto encefálico por embolia aérea. Caso clínico
}

\author{
Patricio Mellado T1, Freddy Constanzo P'1, \\ Juan Francisco Miquel $\mathbf{P}^{2}$, Patricio Ibáñez $\mathbf{L}^{2}$. \\ Ischemic brain infarction \\ after an air embolism. Case report
}

Ischemic stroke due to embolic air is uncommon. There are few reports of patients with air embolic stroke as a complication of endoscopic procedures. The temporal relationship between the stroke and this procedure is the most important clue for the diagnosis. CT scan and MRI of the brain are confirmatory tests. The morbidity and mortality is high. Patients should be hospitalized in a critical care service and treated as soon as possible with oxygen in a pressure camera. We report a 52 years old woman with an ovarian cancer that, during an upper gastrointestinal endoscopy, had a severe alteration of consciousness that did not respond to the use of Flumazenil. A CT scan showed multiple areas of air embolism in the watershed area between anterior and middle right cerebral arteries. A conservative treatment was decided and the patients died 48 hours later (Rev Méd Chile 2005; 133: 453-6).

(Key Words: Anterior cerebral artery; Brain infarction; Intracranial embolism; Middle cerebral artery)

Recibido el 12 de agosto, 2004. Aceptado en versión corregida el 27 de enero, 2005.

${ }^{1}$ Departamentos de Neurología y ${ }^{2}$ Gastroenterología, Facultad de Medicina, Hospital Clínico de la Pontificia Universidad Católica de Chile. Santiago de Chile.

$\mathrm{L}^{\mathrm{a}}$ a embolia aérea corresponde a la oclusión de un vaso sanguíneo por burbujas de aire. Es una complicación grave, aunque infrecuente, en algunos procedimientos clínicos, resultando en una alta morbilidad y eventualmente la muerte ${ }^{1,2}$. La embolia aérea debe ser considerada en el diagnóstico diferencial de cualquier accidente vascular, si se desarrolla durante o después de un procedimiento endoscópico ${ }^{3,4}$. Se debe considerar

Correspondencia a: Dr. Patricio Mellado T. Marcoleta 347, Santiago, Chile. Fax: 56-2-632-6221. E-mail: pmellado@med.puc.cl que los eventos cerebrovasculares no son comunes durante la endoscopia y que su diagnóstico debe ser efectuado precozmente para iniciar su tratamiento específico.

Describimos una paciente que presentó un accidente vascular encefálico debido a una embolia aérea arterial secundaria a un procedimiento endoscópico.

\section{CASO CLÍNICO}

Paciente mujer de 52 años con cáncer de ovario III C (adenocarcinoma mal diferenciado) diagnosticado 2 años antes. Su tratamiento consistió en una histerec- 
tomía total, salpingooforectomía bilateral, omentectomía y resección ileocólica, además de quimioterapia paliativa e ileostomía a permanencia por cuadros de obstrucción intestinal.

Debido a vómitos persistentes de dos semanas de duración, se realizó una endoscopia digestiva alta en forma ambulatoria. Se utilizó $4,5 \mathrm{mg}$ de midazolam intravenoso como sedación. La endoscopia mostró una extensa esofagitis del tercio medio y distal, se tomó biopsias de estas zonas (Figura 1). Durante el procedimiento presentó compromiso de conciencia que no revirtió con flumazenil. En su examen físico general destacaba una frecuencia cardíaca de 100 por min e hipotensión arterial (90/50), que no respondió a la infusión de volumen. En el examen neurológico destacaba: coma y rigidez de descerebración bilateral, ojos desviados a derecha, reflejos oculocefálicos presentes, reflejo corneal abolido a izquierda, hipertonía de las cuatro extremidades, mayor en el hemicuerpo izquierdo, reflejos osteotendíneos aumentados simétricamente, clonus y Babinski bilateral (escala de NIHSS=27). Se decidió su traslado a la sala de reanimación del servicio de urgencias y se solicitó una tomografía computada de encéfalo (TC), la que mostró múltiples embolias aéreas en el territorio limítrofe superficial entre las arterias cerebral media y anterior derechas (Figura 2). La radiografía de tórax fue normal.

El equipo oncológico tratante decidió, en conjunto con la familia de la paciente, un manejo conservador, debido al mal pronóstico de la neoplasia de base, indicándose su traslado a sala y oxígeno al 100\%. La paciente falleció a las $48 \mathrm{~h}$ sin recuperar conciencia ni sus déficits neurológicos. La biopsia del esófago mostró una esofagitis intensa y difusa del tercio medio y distal, la que fue interpretada como secundaria a quimioterapia.

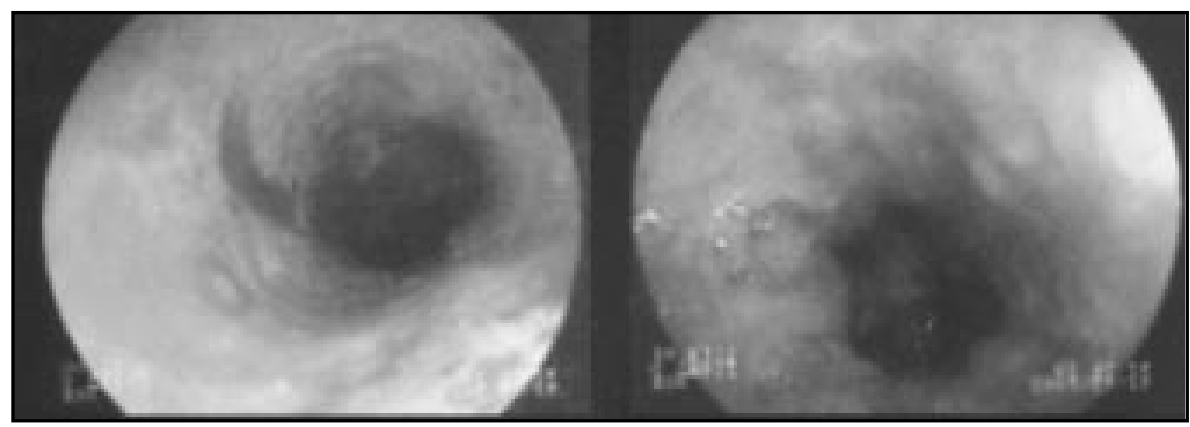

Figura 1. Fotografías del esófago de la paciente que muestran intensa esofagitis difusa del tercio medio y distal.

Figura 2. Tomografía computada de encéfalo. Se observan burbujas de aire en el territorio limítrofe de las arterias cerebrales media y anterior derechas.

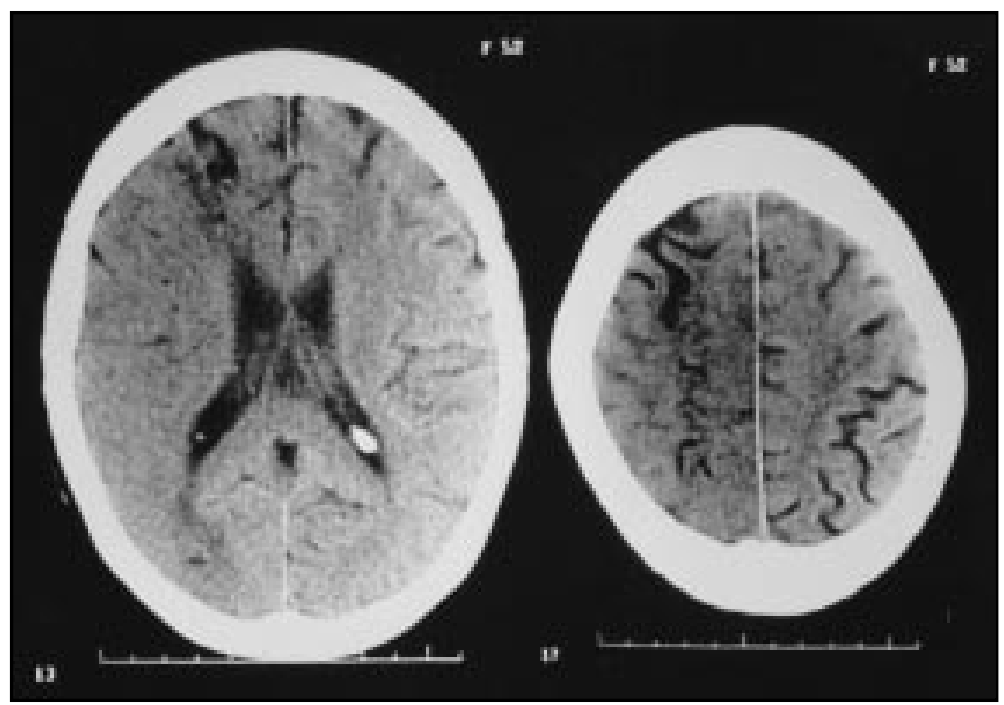




\section{DisCUSIÓN}

El infarto encefálico debido a una embolia aérea es una condición infrecuente, se ha descrito asociada a: trauma, cirugía, cambios bruscos de presión, cateterismo venoso central, ventilación mecánica, biopsia pulmonar, hemodiálisis, angiografía y procedimientos endoscópicos y laparoscópicos ${ }^{1-13}$. La embolia puede ocluir una arteria o rara vez una vena, esto último se observa principalmente como una complicación infrecuente neuroquirúrgica ${ }^{1}$. Sus manifestaciones clínicas son variadas, desde cefalea hasta compromiso de conciencia, déficit focal, convulsiones y muerte ${ }^{1-3,6,13}$. Su morbimortalidad es elevada, una revisión de la literatura de 17 pacientes tratados con terapia hiperbárica mostró que 3 enfermos fallecieron (17,6\%), 6 quedaron con un déficit neurológico invalidante $(35,4 \%)$ y sólo 8 sin secuelas $(47 \%)^{4,13}$.

El mecanismo por el cual una embolia aérea se localiza en el árbol arterial encefálico requiere de dos fenómenos: 1. Entrada de aire al sistema sanguíneo. 2. Paso del aire al circuito izquierdo, es decir, saltándose el pulmón que actúa como un filtro para estas burbujas. Estas burbujas de aire ocluyen arterias de pequeño diámetro (30 a 60 um) y sus consecuencias fisiopatológicas son una reducción en la perfusión distal a la obstrucción y una respuesta inflamatoria por daño endotelial ${ }^{1,3,5,13}$.

La presencia de aire en las arterias sistémicas ha sido explicada por los siguientes mecanismos: sobredistensión pulmonar y barotrauma secundario ${ }^{1-4,7,11,13}$, procedimientos quirúrgicos ${ }^{1-3,5,13}$, instalación de un catéter venoso central ${ }^{11-13}$, hemodiálisis $^{12}$ o infrecuentemente debido a la presencia de fístulas duodeno-cava o esófagovenas pulmonares 2,10 .

El paso de estas burbujas al sistema izquierdo, saltándose el filtro pulmonar, ha sido explicado por embolia paradojal, es decir, el paso de la burbuja desde el sistema venoso al sistema arterial a través de una anomalía vascular, siendo la más frecuente el foramen oval permeable, presente hasta en $30 \%$ de la población ${ }^{1,11,13}$. Otra anomalía vascular que permite esta embolia paradojal es la presencia de un shunt intrapulmonar ${ }^{3,13}$. Por último, como se mencionó, existe la posibilidad del paso directo de aire al sistema izquierdo, por ejemplo en una fístula esófago-vena pulmonar.
Estudios en animales sugieren que grandes embolias aéreas venosas $(20 \mathrm{ml})$ o pequeñas embolias aéreas continuas (11 ml por min) pueden causar embolias arteriales encefálicas ${ }^{1}$. Por otro lado, desde hace ya dos décadas, se utiliza, como un método diagnóstico validado y libre de riesgo, la prueba con microburbujas de aire disuelto en suero fisiológico e inyectadas por vía venosa para la detección de señales de embolia a través de la ecografía Doppler transcraneana, su presencia permite detectar un shunt derechaizquierda. Así, las microburbujas de aire pueden ser utilizadas en la práctica clínica sin riesgo ${ }^{14}$.

En nuestra paciente, al igual que el caso descrito por Weber $\mathrm{M}$ et $\mathrm{al}^{2}$, el mecanismo que hemos postulado es el paso de aire directamente desde el esófago hacia el circuito izquierdo, probablemente hacia las venas pulmonares. Es probable que la esofagitis de la paciente haya facilitado el paso del aire que fue insuflado durante la endoscopia.

El diagnóstico de una embolia aérea encefálica se fundamenta en la presencia de un accidente cerebrovascular relacionado temporalmente con alguno de los factores de riesgo mencionados, siendo indistinguible desde el punto de vista del examen neurológico de otras etiologías del accidente cerebrovascular. La TC o la resonancia magnética de encéfalo son altamente sensibles y específicas para detectar burbujas de aire en el trayecto de los vasos, así como el infarto secundario ${ }^{1-4,6,11}$.

El tratamiento de la embolia aérea encefálica consiste en la estabilización hemodinámica del paciente en una unidad de cuidados intensivos y la oxigenoterapia como su tratamiento específico. Se debe utilizar oxígeno al $100 \%$, o idealmente desde el inicio, cámara hiperbárica ${ }^{1-4,6,11-13}$. La administración de oxígeno no es sólo importante para tratar la hipoxia y la hipoxemia, sino también para eliminar las burbujas de aire en el encéfalo mediante una gradiente de difusión que favorezca su salida ${ }^{1}$. También se ha recomendado la posición supina horizontal, el uso de anticonvulsivantes en forma profiláctica y barbitúricos ${ }^{1}$. La terapia con anticoagulantes y esteroides es controvertida y la mayoría de los autores no la recomienda ${ }^{1}$.

El oxígeno hiperbárico ha sido recomendado como la terapia de primera línea en embolia aérea. Consiste en someter al paciente a presiones barométricas mayores a $760 \mathrm{~mm}$ de $\mathrm{Hg}$, usualmente 
entre 2 y 3 atmósferas por 2 a 4 h, mientras se le administra oxígeno al $100 \%$, lo que disminuye el tamaño de las burbujas por un mecanismo dual: la presión ambiental y la hiperoxia',4,11-15. A 2,8 atmósferas de presión, las burbujas de aire son reducidas al menos en dos tercios de su diámetro inicial $^{12,13}$. La hiperoxia produce una gradiente de difusión dentro de la burbuja de aire, con ingreso de oxígeno a ella y salida de nitrógeno ${ }^{1}$. Además, produce mejoría en el transporte plasmático y la entrega de $\mathrm{O}_{2}$ a los tejidos mal perfundi$\operatorname{dos}^{1,12,13,15}$. Existen reportes de una rápida y completa mejoría neurológica en pacientes que han presentado una embolia aérea encefálica tratada con oxígeno en cámara hiperbárica ${ }^{4,12,13}$. Los mejores resultados se obtienen cuando se inicia la terapia antes de $5 \mathrm{~h}$ del evento, aunque su inicio después de $30 \mathrm{~h}$ puede ser beneficiosa ${ }^{13,16}$.

Nuestra paciente presentó una embolia aérea arterial asociada a una endoscopia digestiva alta, lo

\section{REFERENCIAS}

1. Muth C, Shank E. Gas Embolism. N Engl J Med 2000; 342: 476-82.

2. Weber M, Fiebach J, Lichy M, Weber R, Schwark C, Grau A. Bilateral cerebral air embolism. J Neurol 2003; 250: 1115-7.

3. Akhtar N, Jafri W, Mozaffar T. Cerebral artery air embolism following an esophagogastroscopy: a case report. Neurology 2001; 56: 136-7.

4. Raju G, Bendixen B, Khan J, Summers R. Cerebrovascular accident during endoscopy: consider cerebral air embolism, a rapidly reversible event with hyperbaric oxygen therapy. Gastrointest Endosc 1998; 47: 70-3.

5. Mitchell S, Gorman D. The pathophysiology of cerebral arterial gas embolism. Extra Corpor Technol 2002; 34: 18-23.

6. Hodics T, LiNFante I. Cerebral air embolism. Neurology 2003; 60: 112.

7. Foster P, Boriek A, Butler B, Gernhardt M, Bove A. Patent foramen ovale and paradoxical systemic embolism: a bibliographic review. Aviat Space Environ Med 2003; 74: B1-B64.

8. Katzgraber F, Glenewinkel F, Rittner C, Beule J. Fatal air embolism resulting from gastroscopy. Lancet 1995; 346: 1714-5. que está descrito como una rara complicación de este procedimiento, principalmente cuando es insuflado aire bajo presión por el gastroscopio o cuando se realizan procedimientos como biopsia, proponiéndose como mecanismos la embolia paradójica o la fístula esófago-vena pulmonar ${ }^{1-4,10,17}$. El mecanismo por el cual nuestra paciente presentó una embolia aérea arterial encefálica no fue estudiado debido a su manejo conservador.

\section{CONCLUSIÓN}

El infarto encefálico debido a una embolia aérea es infrecuente, debe sospecharse en todo paciente que desarrolle un déficit neurológico durante o inmediatamente después de un procedimiento invasivo. Su diagnóstico oportuno con una TC o RM de encéfalo puede permitir su tratamiento con oxigenoterapia al $100 \%$ e idealmente en una cámara hiperbárica.

9. Bou-Samra G, Darby P, Christensen G. Cerebral air embolism during endoscopy. Mo Med 1997; 94: 704-7.

10. Christl S, Scheppach W, Peters U, Kirchner T. Cerebral air embolism after gastroduodenoscopy: complication of a duodenocaval fistula. Gastrointest Endosc 1994; 40: 376-8.

11. Emaerel P, Gevers A, De Bruecker Y, Sunaert S, Wilms G. Stroke caused by cerebral air embolism during endoscopy. Gastrointest Endosc 2003; 57: 134-5.

12. Tibbles P, EdelsBerg J. Hyperbaric-oxygen therapy. N Engl J Med 1996; 334: 1642-8.

13. Murphy B, Harford F, Cramer F. Cerebral air embolism resulting from invasive medical procedures. Treatment with hyperbaric oxygen. Ann Surg 1985; 210: 242-5.

14. Meairs S. Hennerici M, Mohr JP. Ultrasonography. En: Mohr JP, Choi DW, Grotta JC, Weir B, Wolf PA (eds). Stroke: Patophysiology, diagnosis, and management. Philadelphia. Churchill Livingstone 2004; 497-524.

15. Pomes J. Oxigenoterapia hiperbárica. En: Cubillos L, Espinoza R. Manual de Urgencias Médicas. Santiago. Mediterráneo 2002; 504-12.

16. Bitterman H, Melamed Y. Delayed hyperbaric treatment of cerebral air embolism. Israel J Med Sci 1993; 29: 22-6.

17. Katzgraber F, Glenewinkel F, Fischler S, Rittner C. Mechanism of fatal air embolism after gastrointestinal endoscopy. Int J legal Med 1998; 111: 154-6. 